\title{
Differential fan effect and attentional focus
}

\author{
MYEONG-HO SOHN, JOHN R. ANDERSON, LYNNE M. REDER, and ADAM GOODE \\ Carnegie Mellon University, Pittsburgh, Pennsylvania
}

\begin{abstract}
As people study more facts about a concept, it takes longer to retrieve a particular fact about that concept. This fan effect (Anderson, 1974) has been attributed to competition among associations to a concept. Alternatively, the mental-model theory (Radvansky \& Zacks, 1991) suggests that the fan effect disappears when the related concepts are organized into a single mental model. In the present study, attentional focus was manipulated to affect the mental model to be constructed. One group of participants focused on the person dimension of person-location pairs, whereas the other group focused on the location dimension. The result showed that the fan effect with the focused dimension was greater than the fan effect with the nonfocused dimension, which is contrary to the mental-model theory. The number of associations with a concept is indeed crucial during retrieval, and the importance of the information seems to be accentuated with attentional focus.
\end{abstract}

The fan effect refers to an increase in response time and/or error rates on a memory test with an increase in the number of competing associations to that memory probe. The associations to a concept were assumed to "fan" out of the concept node, hence the name. Since its first demonstration by Anderson (1974), the fan effect has been replicated in many different experimental paradigms with different types of stimuli (Lewis \& Anderson, 1976; Reder \& Ross, 1983; Zbrodoff, 1995). The assumptions underlying the account of the fan effect specify how and why retrieval processes interact with memory representations. Specifically, multiple facts linked to a concept in the probe will interfere with each other during retrieval because of limited cognitive resources allocated to the probe. As more associations are attached to the probe, the amount of activation that spreads down any path from the probe is reduced, requiring more time for a particular fact to be retrieved. Alternatively, however, there has emerged a competing view that emphasizes a representational account based on situation models (e.g., Radvansky \& Zacks, 1991). In the present study, we seek to incorporate the different views of the fan effect and to test the predictions of these accounts.

In the fan paradigm, participants learn arbitrary associations between concepts (e.g., "Hippie-Park"). In the present study, participants memorized a set of 28 facts about people in locations. Figure 1 shows a basic network representation of some facts and their associated concepts. These facts are constructed so that one, two, or three facts are studied about each person and location. After committing these facts to memory, participants are tested on their ability to recognize person-location pairs previously

This research was supported by National Science Foundation Grant BCS-9975220. We thank Mike Rinck, Ed O'Brien, and Gabe Radvansky for their helpful comments on an earlier version of this article. Correspondence should be addressed to M.-H. Sohn, Department of Psychology, 2125 G. St. NW, 313A Bldg GG, George Washington University, Washington, DC 20052 (e-mail: mhsohn@gwu.edu). studied (targets), and to reject novel combinations of the same people and locations (foils). The fan of a probe is the number of facts associated with the person and the location, and the reaction latency increases with the fan. The fan effect holds for both targets and foils, although sometimes the effect size varies (Anderson, 1976).

The present study is concerned with better understanding why the size of the fan effect for different dimensions of the stimuli (e.g., person vs. location) sometimes varies and what influences that variation. In some studies, the size of the fan effect is comparable for both dimensions (Anderson, 1974). However, some types of material have produced different size fan effects for different dimensions (Radvansky \& Zacks, 1991), the phenomenon known as the differential fan effect. First, we will describe the mentalmodel theory that was initially proposed to explain the differential fan effect. Second, we will describe the ACT-R theory, and how it differs from the mental-model theory.

According to mental-model theory, facts are organized into mental models when the material is studied. For example, when object-location pairs are studied (e.g., "The potted plant is in the hotel"), these associations should be organized into location-based mental models because a location can have many items in it, but an object can be in only one place at a time. Therefore, a location contains all the associated objects in its own mental model, whereas an object may appear in multiple location-based mental models. In this example, locations organize the mental models, serving as the organizing dimension. According to Radvansky and Zacks (1991), there is only a fan effect of the nonorganizing dimension, since this will result in more mental models and it takes time to search through these: The fan of the organizing dimension will have no effect because the objects within a mental model can be accessed quickly with little effect of the number of objects. In accordance with the hypothesis, the results showed that the location fan effect was smaller than the object fan effect (Radvansky \& Zacks, 1991). 


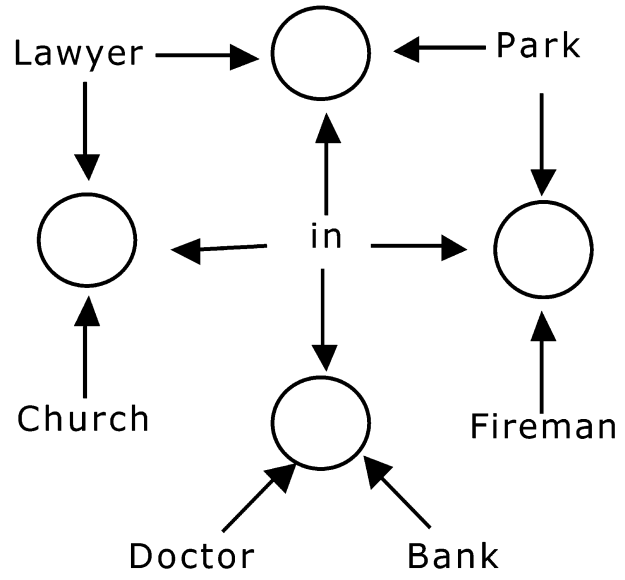

Figure 1. An illustration of network representation of facts and their associated concepts.

The mental-model theory has been generalized to other materials (Radvansky, Spieler, \& Zacks, 1993; Radvansky, Wyer, Curiel, \& Lutz, 1997). Radvansky et al. (1993) argued that person-location pairs follow different organizing principles, depending on the relative size of locations. With relatively large locations (e.g., public library, hotel), either type of mental model can be formed because people go to many places, and a place can contain many people. With relatively small locations (e.g., telephone booth, tanning bed), person-based mental models are to be formed because these small places can only contain one person at a time, whereas the same person can go to all of these places. This analysis was used to explain why the person fan effect was smaller than the location fan effect with relatively small locations, whereas the differential fan effect was not obtained with relatively large locations.

In contrast, ACT-R emphasizes the retrieval processes. In ACT-R, activation spreads from presented terms to the connected nodes that represent various facts (Figure 1). The latency to retrieve any fact from memory is determined by activation level of that fact. The activation, $A_{i}$, of a particular fact $i$ is determined by the following activation equation:

$$
A_{i}=B_{i}+\sum_{j} W_{j} S_{j i}
$$

where $B_{i}$ is the base-level activation of the fact, reflecting its recency and frequency of study. The summation is over the fact concepts, $j$, which are the activation sources when the probe is presented for recognition. In the present study, these sources are person, location, and the preposition in. The multiplier, $W_{j}$, is the amount of attention given to a dimension to which a particular source $j$ belongs. In ACT-R, the fan effect depends on the associative strength, $S_{j i}$, which is the relative associative strength of the fact $i$ to a given concept $j$. As more facts are associated with a concept, the associative strength of a particular fact is weakened because of competition for limited resources. If there is no factor biasing toward person or location, the source activation is assumed to be divided equally between the person and the location dimensions. This is reflected in equal values for $W_{j}$. However, if for some reason either dimension is emphasized more than the other during learning, that dimension may get a higher attentional weight (Anderson \& Reder, 1999). Consequently, as implied in the activation equation, the dimension with higher attentional weight will produce a greater fan effect than the dimension with lower weight.

In summary, the mental-model and ACT-R theories offer rather different conceptions of what produces the differential fan effect. According to ACT-R, the dimension that gets emphasized has a higher attentional weighting and should have the greater fan effect. So, for example, when there is a larger fan effect of the object dimension than the location dimension (e.g., Radvansky \& Zacks, 1991), it is not because the location is the organizing dimension, as the mental-model theory would argue. Rather, it is because the object is the emphasized dimension. Thus, the two theories attribute the same effect to special processing of different dimensions.

The problem with past research is that there has been no explicit manipulation to influence which dimension receives special processing. Past research has typically taught participants the material with a dropout procedure in which they have to produce the correct response to probes of the form "Who is in this location?" or "Where is this person?" This paradigm ensures that participants receive questions anchored on both location and person, with the intention of emphasizing both dimensions equally. Despite probing from both terms during study, participants sometimes seem to organize the information around just one term (e.g., Radvansky \& Zacks, 1991). It was inferred that this was due to how participants would organize the material, but this choice was not directly manipulated.

The present study manipulated the way participants organize the person-location material during the study phase. Participants in the person-focus condition received only person-anchored questions, with the explicit instruction to organize material into person models. These models involve each person visiting multiple locations (e.g., "The lawyer was in the airport, the stadium, and the classroom"). Conversely, participants in the location-focus condition received only location-anchored questions and were instructed to organize the material into location models. In this case, the model involves locations that contain multiple people (e.g., "In the stadium, there was the lawyer, the senator, and the grandma"). Both groups received the same person-location pairs - only the manner in which the material was studied differed. Presenting one type of cued recall question during learning was necessary to manipulate the dimension that would be the focus of attention, or the organizing dimension for mental models. The mental-model account predicts that the fan effect of the organizing dimension should be smaller than that of the nonfocused dimension. However, the ACT-R theory makes the opposite prediction. 
Table 1

Mean Latency (RT, in Milliseconds) and Accuracy

\begin{tabular}{|c|c|c|c|c|c|c|c|c|c|c|c|c|}
\hline \multirow{3}{*}{$\begin{array}{l}\text { Nonfocused } \\
\text { Dimension }\end{array}$} & \multicolumn{12}{|c|}{ Focused Dimension } \\
\hline & \multicolumn{4}{|c|}{$\begin{array}{c}\text { Target } \\
\text { Fan } 2 \\
\end{array}$} & \multicolumn{2}{|c|}{ Fan 3} & \multicolumn{2}{|c|}{ Fan 1} & \multicolumn{2}{|c|}{$\begin{array}{c}\text { Foil } \\
\text { Fan } 2\end{array}$} & \multicolumn{2}{|c|}{ Fan 3} \\
\hline & RT & Acc. & RT & Acc. & RT & Acc. & RT & Acc. & RT & Acc. & RT & $\overline{\text { Acc. }}$ \\
\hline \multicolumn{13}{|c|}{ Person-Focus Condition } \\
\hline Fan 1 & 1,110 & .96 & 1,184 & .97 & 1,298 & .94 & 1,273 & .96 & 1,358 & .96 & 1,479 & .93 \\
\hline Fan 2 & 1,226 & .95 & 1,280 & .91 & 1,424 & .86 & 1,418 & .93 & 1,467 & .88 & 1,542 & .88 \\
\hline Fan 3 & 1,186 & .95 & 1,364 & .87 & 1,508 & .79 & 1,384 & .91 & 1,500 & .87 & 1,517 & .83 \\
\hline \multicolumn{13}{|c|}{ Location-Focus Condition } \\
\hline Fan 1 & 1,057 & .94 & 1,174 & .91 & 1,208 & .86 & 1,243 & .91 & 1,307 & .91 & 1,443 & .94 \\
\hline Fan 2 & 1,064 & .95 & 1,294 & .92 & 1,414 & .84 & 1,239 & .97 & 1,399 & .83 & 1,505 & .93 \\
\hline Fan 3 & 1,181 & .96 & 1,458 & .88 & 1,448 & .85 & 1,303 & .96 & 1,409 & .96 & 1,572 & .92 \\
\hline
\end{tabular}

\section{METHOD}

\section{Participants}

There were two groups of participants, one assigned to the personfocus condition (19 participants) and another to the location-focus condition (17 participants). Participants were undergraduate students at Carnegie Mellon University. They participated in return for monetary reward.

\section{Procedure}

In the study phase, participants were presented with 28 sentences regarding a person in a location. To accentuate the representational difference, we used photographs in the person-focus condition and a spatial layout in the location-focus condition. In the person-focus condition, participants viewed 25 photographs of individuals they did not know (graduate students and staff members of the psychology departments of Carnegie Mellon University and the University of Pittsburgh, 12 Caucasian male and 13 Caucasian female, age range 20-30). For each participant, 17 of these pictures were randomly assigned to occupation names such as lawyer and senator. A personlocation sentence (e.g., "The lawyer is in the park") was presented with the picture of the corresponding person. Occupation names and places are presented in the Appendix. After reading the sentences with the pictures, participants went through a two-tiered questionanswering phase. First, they were presented with 25 faces in a $5 \times 5$ grid and were asked to identify the correct face (e.g., "Click on the lawyer"). If a wrong face was identified, the participant was prompted with the correct face. After identifying the person correctly, participants were asked to type in all the places associated with that person. Wrong answers were corrected. To prevent participants from using a location-based strategy, each time a new question was asked, the 25 faces were presented in a new random configuration of the $5 \times 5$ grid.

In the location-focus condition, participants were presented with a $5 \times 5$ grid. From the 25 possible positions in the grid, 17 were randomly selected for each participant and were assigned location names such as park and church. A sentence was presented in the grid cell that was assigned to the corresponding location. Participants were instructed to memorize which location was at which grid position, and who were the occupants. In the question-answering phase, the participants were also presented with two-fold questions. First they were asked to identify a certain location among 25 grids (e.g., "Click on the park"). When the location was correctly identified, they were asked to type all the people associated with the location.

Participants' memory for the associations was strengthened with a two-pass dropout cued recall procedure. In each pass, participants received two-fold questions as described above in a random order of all possible questions. If they could not answer correctly, the question was repeated after all the other questions had been asked. This continued until all questions had been answered correctly twice.
During the recognition test, participants judged 168 targets and 168 foils in the form of a person-location pair ("lawyer-park"). The order of the person and location within the probe was randomized. To create foils, we swapped person-location pairs from the same fan condition. The probes were presented in 6 blocks of 56 trials. On every trial, a fixation was presented for $500 \mathrm{msec}$. The probe was presented immediately and remained on the screen until the participant's response was made. The feedback on accuracy and speed was presented for $3 \mathrm{sec}$.

\section{RESULTS}

A four-way analysis of variance was conducted for latency and accuracy with attentional focus (person or location), focused dimension (three fans), nonfocused dimension (three fans), and trial type (target and foil) as variables. The attentional focus was a between-groups factor.

For the latency analysis, only correct trials with latency shorter than $3 \mathrm{sec}$ were included. Less than $7 \%$ of trials were eliminated because of excessively long responses. Mean accuracy and latency, presented in Table 1, were negatively correlated across the various conditions defined by the combinations of the variables $(r=-.56, p<$ .01 ), indicating that conditions with poor performance by one measure had poor performance by the other. Table 2 presents a detailed report of the significant effects involving accuracy and latency. One may notice a couple of significant interactions involving nonfocused dimension and trial type. In accuracy, the interaction between nonfocused fan and trial type was significant. The nonfocused fan effect was significant for targets $\left[F(2,70)=36.08, M S_{\mathrm{e}}=\right.$ $.002, p<.0001]$, but not for foils $(p>.70)$. Also, in latency, a three-way interaction was significant, involving attentional focus, nonfocused fan, and trial type. In the person-focus condition, the interaction between nonfocused fan and trial type was not significant $(p>.20)$. In the location-focus condition, the nonfocused fan effect was significant for targets $\left[F(2,16)=32.91, M S_{\mathrm{e}}=6,723\right.$, $p<.0001$ ], but not for foils $(p>.20)$. It is not clear at this point why the nonfocus fan effect differs depending on trial type. Below we report the main result of this study, the differential fan effect in latency.

The latency of each condition is plotted in Figure 2. There was a significant fan effect both for the emphasized 
Table 2

Summary of Significant Results

\begin{tabular}{lc}
\hline \multicolumn{1}{c}{ Conditions } & \multicolumn{1}{c}{ Statistics } \\
\hline Focused fan & Accuracy \\
Nonfocused fan & $F(2,68)=21.76, M S_{\mathrm{e}}=.01, p<.0001$ \\
Attentional focus $\times$ focused fan & $F(2,68)=10.46, M S_{\mathrm{e}}=.007, p<.0001$ \\
Focused fan $\times$ nonfocused fan & $F(2,68)=4.99, M S_{\mathrm{e}}=.01, p<.01$ \\
Nonfocused fan $\times$ trial type & $F(4,136)=3.33, M S_{\mathrm{e}}=.008, p<.05$ \\
& $F(2,68)=13.38, M S_{\mathrm{e}}=.008, p<.0001$ \\
Focused fan & Latency \\
Nonfocused fan & $F(2,68)=56.80, M S_{\mathrm{e}}=47,356, p<.0001$ \\
Trial type & $F(2,68)=30.91, M S_{\mathrm{e}}=36,066, p<.0001$ \\
Nonfocused fan $\times$ trial type & $F(2,68)=94.29, M S_{\mathrm{e}}=36,066, p<.0001$ \\
Attentional focus $\times$ focused fan $\times$ trial type & $F(2,68)=5.64, M S_{\mathrm{e}}=19,105, p<.01$ \\
& $F(2,68)=4.18, M S_{\mathrm{e}}=18,128, p<.05$ \\
\hline
\end{tabular}

or organizing dimension and for the nonfocused dimension. The critical issue is the relative size of the fan effect for these dimensions. The mental-model theory predicts a smaller fan effect for the focused dimension than for the nonfocused dimension, while the ACT-R theory predicts the opposite. To test this, the fan slope was calculated for the focused and the nonfocused conditions. The slope was defined as the mean latency increase when there is an increase of the fan for a particular dimension. In the personfocus condition, the fan effect of the focused dimension (person dimension, $98 \mathrm{msec}$ ) was greater than that of the nonfocused dimension [location dimension, $63 \mathrm{msec}$; $t(18)=2.59, p<.05]$. In the location-focus condition, the fan effect of the focused dimension (location dimension, $118 \mathrm{msec}$ ) was greater than that of the nonfocused dimension [person dimension, $73 \mathrm{msec} ; t(16)=2.80, p<$ $.05]$. The differential fan effect (i.e., the difference between the fan effects of the two dimensions) in each condition did not differ from each other $(p>.50)$. The fan effect of the focused dimension is comparable to those obtained in other studies using similar materials and designs (e.g., Anderson, Bothell, \& Douglass, 2004). As ACT-R suggests, regardless of the type of mental model that was supposed to be constructed following the instruction, the focused dimension resulted in a greater fan effect than did the nonfocused dimension. This result supports ACT-R in that the focused dimension received higher attentional weight than the nonfocused dimension.

\section{MODELING}

Previously developed ACT-R models for the fan effect (Anderson \& Lebiere, 1998; Anderson \& Reder, 1999) were used to simulate the differential fan effects obtained in the present study. These models assume that a participant begins by encoding the person and the location terms into the problem representation, then retrieves the relevant personlocation fact, compares the retrieved result with the problem representation, and finally executes the response. The timing of the encoding, comparison, and motor execution are considered as intercepts and the current modeling effort concentrates on the retrieval time. In ACT-R, the activation level of information determines the retrieval time according to the following latency equation:

$$
T=F e^{-A},
$$

where $F$ is the scale factor, which is to be estimated. In the activation equation given earlier, the total activation is a sum of base level activation $(B)$ and the sum of weighted associative strengths $\left(W_{j} S_{j i}\right)$. The associative strength $\left(S_{j i}\right)$

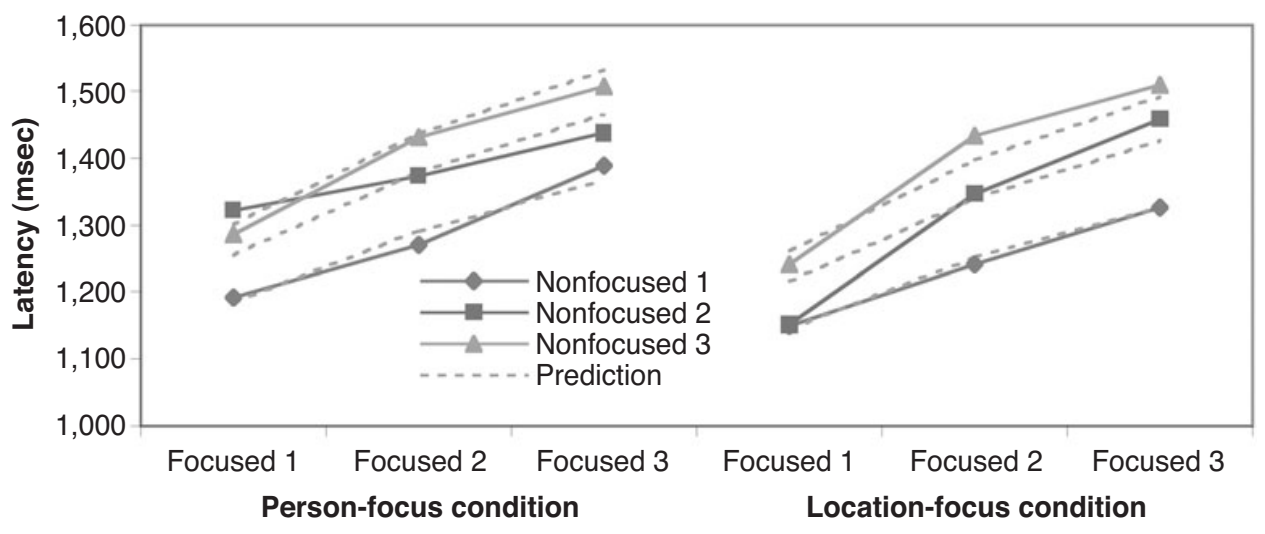

Figure 2. Latency as a function of focused fan, nonfocused fan, and trial type in each attentional focus condition. 
depends on the total associative strength $(S)$ and the number of associations from the concept, and is expressed as $S-\ln (n)$, where $n$ is the number of associations and $S$ is estimated. The attentional weight, whose total amount is fixed at 1 , is the critical parameter because ACT-R predicts that the dimension with a higher attentional weight should result in a greater fan effect. In previous ACT-R models with no emphasis on a particular dimension, it was assumed that the total amount of $W$ is divided equally among three terms - two dimensions of facts and the preposition. Therefore, .667 is the amount of activation to be distributed between the person and location dimensions in this experiment, and we estimate what amount of this is given to the organizing dimension. In sum, five parameters were estimated, including the three parameters common to both conditions: scale factor $(F)$, associative strength $(S)$, and attentional weight for the organizing dimension $\left(W_{f}\right)$. Note that the activation and the latency equation predict only retrieval times of fan facts. To perform a task, other processes are also necessary, such as encoding a stimulus and producing a response. These times are considered as intercepts. Different intercepts $(I)$ were estimated for each condition to reflect the differences between groups. These five parameters were estimated, while minimizing the $\chi^{2}$ deviation of the fit to the data defined as

$$
\sum_{i}\left(\hat{X}_{i}-\bar{X}_{i}\right)^{2} / s_{\bar{x}}^{2}
$$

which is the ratio of the actual deviation (sum of the squared deviations of the predicted means from the actual means) to the estimated means (squared standard errors obtained from the participant-by-condition interaction). Therefore, a significant $\chi^{2}$ deviation indicates a poor fit of the model to data. This is a statistic whose degrees of freedom is the number of observations (36 in total) minus the number of parameters (5). These predictions are displayed along with the data in Figure 2, and the parameter values are presented in Table 3. This intercepts are different between different attentional focus conditions, reflecting the between-groups difference. The ACT-R model fits the data quite well, estimating the same parameters for both attentional focus conditions $\left[\chi^{2}(31)=38.78, p>.1\right]$. Moreover, the estimated $W_{f}(.398)$ is greater than the attentional weight of the nonfocused dimension $\left(W_{n}=.269\right)$. Alternatively, we fit the data with an equal amount of $W$ for both dimensions (.334), which produced a significant deviation from the data $\left[\chi^{2}(32)=48.78, p<.05\right]$. This strongly supports the view that attentional focus can shift the amount of ac- tivation that is sent from one dimension or another, and the dimension with greater emphasis produces a greater fan effect.

\section{DISCUSSION}

The purpose of the present study was to examine the effect of memory representation on memory retrieval. We essentially forced participants into using one of two representations by requiring them to answer questions anchored on only one of the dimensions (location or person). The fan effect of the focused dimension was significantly greater, which is inconsistent with the mental-model theory. How, then, are we to understand the results that have been regarded as supporting the mental-model theory of the differential fan effects (e.g., Radvansky \& Zacks, 1991)? With object-location pairs, for example, a substantial fan effect of the object dimension was obtained, but no fan effect of the location dimension was obtained. This result was interpreted as participants forming location-based mental models. Given the results of the present study, however, it would seem that the object dimension, not the location dimension, served as an organizing dimension when the fan effect was greater for the object dimension.

One difference between our study and those of Radvansky and his colleagues is that we manipulated the focus of attention to bias which dimension should be selected as the organizing dimension. In their studies, the organizing dimension was assumed to have emerged naturally on the basis of the semantic information implied by the materials. One might wonder whether attending to a certain dimension is sufficient to allow organization of information around that dimension. Zwaan, Radvansky, Hilliard, and Curiel (1998) varied whether spatially organized information was emphasized or not. They found that reading times increased when the relevant locations shifted within text, only when spatial information was emphasized. However, this effect disappeared when the spatial information was not emphasized. This result seems consistent with our assumption that the attended dimension may serve as an organizing dimension.

The mental-model class of theories has been quite successful and influential in many cognitive domains, such as reasoning (Johnson-Laird, 1983), spatial thinking (Taylor \& Tversky, 1996), text comprehension (Bower \& Rinck, 2001), complex problem solving (Kintsch, 1988), and so forth. The present study is concerned with a particular application of the mental-model view on memory retrieval

Table 3

Parameter Values and the Result of the Best-Fitting ACT-R Model

\begin{tabular}{|c|c|c|c|c|c|c|}
\hline & \multicolumn{4}{|c|}{ Parameters } & \multicolumn{2}{|c|}{ Model Fit } \\
\hline & $I$ & $F$ & $W_{f}$ & $S$ & $\chi^{2}$ & $d f$ \\
\hline Person-focus condition & $689 \mathrm{msec}$ & & & & & \\
\hline Location-focus condition & $650 \mathrm{msec}$ & $911 \mathrm{msec}$ & 0.398 & 1.25 & 38.78 & 31 \\
\hline
\end{tabular}

Note-I, intercept. 
proposed by Radvansky and his colleagues (Radvansky \& Zacks, 1991; Radvansky et al., 1993; Radvansky et al., 1997). Therefore, implications of the present study for the mental-model view are limited. However, it is noteworthy that the memory representation, which lies in the core of the mental-model view in general, can be successfully incorporated into an information-processing model that emphasizes retrieval processes. As Radvansky (1999) pointed out, memory representation may be incorporated into the ACT-R framework as part of declarative knowledge, which is the long-term memory for ACT-R. We would add that the organization of declarative knowledge reflects the attentional focus given to different aspects of information.

In conclusion, two implications of ACT-R for memory retrieval have been supported. First, the strength of the association between a concept and a fact in memory is adjusted to reflect fan. Second, it is possible to vary the weighting given to various types of concepts by emphasizing one of the concepts. The ACT-R theory predicts larger fan effects for concepts that receive greater attention; empirical data and computational modeling of these data have supported this prediction.

\section{REFERENCES}

ANDERSON, J. R. (1974). Retrieval of propositional information from long-term memory. Cognitive Psychology, 5, 451-474.

ANDERSON, J. R. (1976). Language, memory, and thought. Hillsdale, NJ: Erlbaum.

Anderson, J. R., Bothell, D., \& Douglass, S. (2004). Eye movements do not reflect retrieval processes: Limits of the eye-mind hypothesis. Psychological Science, 15, 225-231.
Anderson, J. R., \& Lebiere, C. (EDs.) (1998). The atomic components of thought. Mahwah, NJ: Erlbaum.

ANDERSON, J. R., \& REDER, L. M. (1999). The fan effect: New results and new theories. Journal of Experimental Psychology: General, $\mathbf{1 2 8}$, 186-197.

BowER, G. H., \& RINCK, M. (2001). Selecting one among many referents in spatial situation models. Journal of Experimental Psychology: Learning, Memory, \& Cognition, 27, 81-98.

JoHnson-Laird, P. N. (1983). Mental models. Cambridge: Cambridge University Press.

KINTSCH, W. (1988). The role of knowledge in discourse comprehension: A construction-integration model. Psychological Review, 95, 163-182.

LEWIS, C. H., \& ANDERSON, J. R. (1976). Inferences with real world knowledge. Cognitive Psychology, 8, 311-335.

Radvansky, G. A. (1999). The fan effect: A tale of two theories. Journal of Experimental Psychology: General, 128, 198-206.

Radvansky, G. A., Spieler, D. H., \& Zacks, R. T. (1993). Mental model organization. Journal of Experimental Psychology: Learning, Memory, \& Cognition, 19, 95-114.

Radvansky, G. A., Wyer, R. S., JR., Curiel, J. M., \& Lutz, M. F. (1997). Situation models and abstract ownership relations. Journal of Experimental Psychology: Learning, Memory, \& Cognition, 23, 12331246.

RAdVANSKY, G. A., \& ZaCKs, R. T. (1991). Mental models and the fan effect. Journal of Experimental Psychology: Learning, Memory, \& Cognition, 17, 940-953.

REDER, L. M., \& Ross, B. H. (1983). Integrated knowledge in different tasks: The role of retrieval strategy on fan effects. Journal of Experimental Psychology: Learning, Memory, \& Cognition, 9, 55-72.

Taylor, H. A., \& Tversky, B. (1996). Perspective in spatial descriptions. Journal of Memory \& Language, 35, 371-391.

ZBrodofF, N. J. (1995). Why is $9+7$ harder than $2+3$ ? Strength and interference as explanations of the problem-size effect. Memory \& Cognition, 23, 689-700.

Zwaan, R. A., Radvansky, G. A., Hilliard, A. E., \& Curiel, J. M. (1998). Constructing multidimensional situation models during reading. Scientific Studies of Reading, 2, 199-220.

APPENDIX

Concepts Used in the Study

\begin{tabular}{|c|c|}
\hline Person & Location \\
\hline $\begin{array}{l}\text { banker, beggar, bishop, cowboy, cyclist, } \\
\text { dancer, grandma, grocer, hippie, infant, } \\
\text { maiden, mermaid, prince, prowler, } \\
\text { senator, soldier, speaker, surgeon, } \\
\text { tourist, typist, vandal, writer }\end{array}$ & $\begin{array}{l}\text { airport, capitol, college, desert, factory, } \\
\text { kitchen, library, museum, prison, resort, } \\
\text { rocket, stable, stadium, subway, tavern, } \\
\text { taxicab, theater, trailer, tunnel, valley, } \\
\text { village, winery }\end{array}$ \\
\hline
\end{tabular}

Note-For each participant, 17 concepts were selected from each category to make up the fan materials. 